\title{
DESARROLLO DEL PENSAMIENTO LÓGICO BASADO EN RESOLUCIÓN DE PROBLEMAS EN NIÑOS DE 4 A 5 AÑOS
}

\author{
Development of logical thinking based on problem solving in children of 4 to 5 years
}

Desenvolvimento do pensamento lógico baseado em resolução de problemas em crianças de 4 a 5 anos

RECIBIDO:11DE ABRILDE 2016

Ana Patricia León Urquijo (Colombia)

apleonurquijo@hotmail.com

Corporación Universitaria Minuto de Dios

Doctora en Filosofía y Ciencias de la

Educación

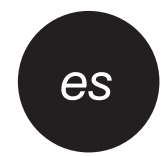

\section{RESUMEN}

Se presenta los resultados de la investigación descriptiva longitudinal panel "Desarrollo del pensamiento lógico matemático basado en resolución de problema en niños y niñas de 4 a 5 años" de una institución educativa pública de Osorno, Chile, que se realizó mediante un programa de intervención educativa basada en problemas durante 2013-2014, utilizando estrategia didáctica de la vida práctica a través del juego, con el propósito de analizar los procesos del desarrollo del pensamiento lógico. El instrumento fue la "Escala para Observar Estrategias de Resolución de Problemas" de Sáiz y Román (2011). Además se hizo el registro de notas de campo. En este artículo se presentan los resultados de los ítems de atención comprensión y motivación. Se encontró que algunos niños son ingeniosos buscando recursos para resolución de situaciones problemas, otros imitan, piden ayuda y resuelven con la repetición de instrucciones; algunos necesitan apoyo concreto con sugerencias y muy pocos no se esfuerzan ni intentan hacerlo. Se concluyó que el desarrollo del pensamiento se propicia en un ambiente social donde la educadora juega un papel importante contribuyendo a la interacción de los niños haciendo uso de la comunicación efectiva y apoyo mutuo.

PALABRAS CLAVE: aprendizaje, atención, comprensión, lógico, motivación, pensamiento, problemas.
EVALUADO:19DEAGOSTODE 2016

Jacqueline del Carmen Casas Antilef (Chile)

Jakelin.casas@gmail.com

Universidad de los Lagos

Magister en Ciencias de la Educación

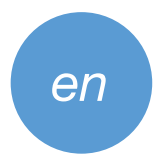

ABSTRACT

We present the results of the descriptive longitudinal panel "Development of mathematical logical thinking based on problem solving in children aged 4 to 5 years" from a public educational institution in Osorno, Chile. It was carried out through an educaproblems during 2013-2014, using didactic the purpose of analyzing the processes of the development of logical thinking. The instrument used was the "Scale to Observe Strategies for Problem Solving" by Sáiz y Román (2011). In addition, field notes were registered. In this paper, we present the results of attention, understanding and motivation items. Some children were found to be resourceful for solving problem situations, while others imitate, ask for help, and resolve them with the repetition of the instructions; tions, and very few do not try or aim to resolve them. It was concluded that the development of the thought is propitiated in a social environment where the educator plays an important role contributing to the interaction of children making use of effective communication and mutual support. Gerardo Restrepo Ramírez (Colombia)
Gerardo.Restrepo@USherbrooke.ca
Profesor Universidad de Sherbrooke
Doctor en Educación tional intervention program based on strategy of practical life through play, with some need concrete support with sugges-

\section{RESUMO}

Se apresenta os resultados da pesquisa descritiva longitudinal painel "Desenvolvimento do pensamento lógico matemático baseado em resolução de problema em meninos e meninas de 4 a 5 anos" de uma instituição educativa pública de Osorno, Chile, que se realizou mediante um programa de intervenção educativa baseada em problemas durante 2013 -2014, utilizando estratégia didática da vida prática através do jogo, com o propósito de analisar os processos do desenvolvimento do pensamento lógico. O instrumento foi a "Escala para Observar Estratégias de Resolução de Problemas" de Sáiz e Román (2011). Além disso fez-se o registro de notas de campo. Em este artigo se apresentam os resultados dos itens de atenção compreensão e motivação. Encontrou-se que alguns meninos são engenhosos procurando recursos para resolução de situações problemas, outros imitam, pedem ajuda e resolvem com a repetição de instruções; alguns necessitam apoio concreto com sugestões e muito poucos não se esforçam nem intentam fazê-lo. Concluiu-se que o desenvolvimento do pensamento se propicia em um ambiente social onde a educadora joga um papel importante contribuindo à interação dos meninos fazendo uso da comunicação efetiva e apoio mútuo.

KEYWORDS: learning, attention, understanding, logical, motivation, thinking, problems.
PALAVRAS CHAVE: aprendizado, atenção, compreensão, lógico, motivação, pensamento, problemas. 
Desde la más temprana edad, los seres humanos dan señales progresivas del desarrollo del pensamiento lógico. Basándose en diversos estudios, Gelman y Gallistel (1978) demostraron que los niños desarrollan capacidades que les permiten acceder al conocimiento numérico que los dirige al posterior aprendizaje de la habilidad matemática. En otras palabras, al llegar al jardín infantil, los niños llevan consigo una serie de competencias matemáticas que les permite desenvolverse en forma concreta resolviendo situaciones en las que demuestran de forma lógica las capacidades que han adquirido en el medio natural de su núcleo escolar, familiar y social, pero que con la ayuda de los educadores amplían, desarrollan, potencian nuevos aprendizajes $\mathrm{y}$ fortalecen los adquiridos.

El desarrollo del pensamiento lógico ocupa un lugar importante en el proceso evolutivo de los niños, porque este conlleva procesos de observación, interpretación, análisis, motivación y comprensión de relaciones, lo que manifiesta diversos principios metacognitivos. A continuación se presenta los resultados de un estudio realizado en la Maestría de Ciencias de la Educación de la Universidad de los Lagos, cuyo objetivo fue describir el proceso de la resolución de problemas matemáticos en los niños de transición I (4 a 5 años), durante el desarrollo de un programa de relación lógico matemáticas y cuantificación con estrategia didáctica de resolución de problemas a través del juego, (como resultado parcial del proyecto "Discursos y prácticas de los profesores de maternal (preescolar) y de primer año: un estudio comparativo entre Quebec-Chile-Colombia"1), que permitieron conocer cómo es el desenvolvimiento de diferentes aspectos de la vida cotidiana al momento de enfrentarse a problemáticas e indaga cómo logran encontrar la solución más adecuada a un problema, o simplemente si optan por utilizar la estrategia más recurrente, considerando las relaciones de la educadora con los niños, estímulos del medio en que se encuentren y los aprendizajes previos que poseen.

1 Proyecto que inició en 2013 y finalizó en 2015, liderado por PhD. Michèle Venet de la Universidad de Sherbrooke (Canadá), desarrollado conjuntamente con la Universidad de los Lagos (Chile) y Universidad Tecnológica de Pereira (Colombia).
Para abordar el estudio se seleccionó una muestra intencionada de 20 niños con edad de 4 a 5 años, de un jardín infantil estatal de Osorno, Chile. Para poder efectuar la intervención educativa se solicitó autorización y consentimiento informado a la institución educativa y el asentamiento informado de los padres o tutores para la participación de los niños en este estudio. El enfoque de estudio fue mixto (cualitativo y cuantitativo) de alcance descriptivo, con diseño metodológico longitudinal panel (Hernández, Fernández y Baptista, 2010), para recolectar y describir los aspectos necesarios como detalles de situaciones de juego y de tareas, interacciones entre los niños y con los adultos y reacción en los juegos ante las problemáticas presentadas, que se registraron durante el año escolar de 2013 -2014.

El instrumento se construyó tomando los ítems (atención, comprensión, motivación, procesamiento de la información, formas de resolver-metacognición, formas de resolver-razonamiento y salida de la información), de "Escala para Observar Estrategias de Resolución de Problemas" de Sáiz y Román (2011). En este artículo se presenta los resultados de los tres primeros ítems correspondientes a atención, comprensión y motivación con sus respectivos indicadores.

Se comparó el diagnóstico con la evaluación final, por medio de la comparación de medias aritméticas con la prueba T Student, la probabilidad o significancia representada por $\alpha=0,05$, con la fórmula estadística:

$$
s_{\mathrm{w}}=\sqrt{\frac{\sum\left(x_{i}-\bar{x}\right)^{2} \cdot n_{i}}{n}}=\sqrt{\frac{\sum x_{i}^{2} \cdot n_{i}}{n}-\bar{x}^{2}}
$$

Los resultados se presentaron en tablas obtenidas mediante el procesamiento de la información en el paquete estadístico SPSS versión 20 (IBM Corporation, 2011), que representa la frecuencia $F$, diagnóstico $\mathrm{D}$, evaluación final $\mathrm{E}$, la media $\mathrm{M}$, número niños $\mathrm{N}$, desviación típica $\mathrm{D}$ tip. y significancia bilateral Sig. (b). Se complementó el análisis de la información con los registros de grabaciones de audio y video, los que reflejan elementos descriptivos de los comportamientos (evidencias verbales y no verbales), tal y como han ocurrido los procesos de resolución de problemas en las interacciones que realizaban los niños en sus procesos de aprendizaje.
| Panorama I pp.98-107 I Volumen 10 I Número 19 I Julio-diciembre | 2016 


\section{ANÁLISIS Y DISCUSIÓN DE LOS RESULTADOS DEL PRO- CESO DEL DESARROLLO DEL PENSAMIENTO LÓGICO A PARTIR DE LA RESOLUCIÓN DE PROBLEMAS}

Ana Patricia

León Urquijo I

Jacqueline del

Carmen Casas

Antilef |

Gerardo

Restrepo

Ramírez |

\begin{abstract}
tegias de resolución de problemas que utilizaron para
\end{abstract} poder desarrollarlos.

\section{ATENCIÓN}

Tabla 1

\begin{tabular}{|c|c|c|c|c|c|c|}
\hline \multicolumn{3}{|c|}{$\begin{array}{l}\text { Mira a la educadora cuando } \\
\text { le pide información }\end{array}$} & \multicolumn{2}{|c|}{$\begin{array}{l}\text { Mira a la edu- } \\
\text { cadora cuando } \\
\text { le propone una } \\
\text { actividad }\end{array}$} & \multicolumn{2}{|c|}{$\begin{array}{l}\text { Sigue órdenes } \\
\text { instrucciones }\end{array}$} \\
\hline & $\mathrm{D}$ & E f & D & $\mathrm{Ef}$ & D & E f \\
\hline & $\mathrm{F}$ & $\mathrm{F}$ & $\mathrm{F}$ & $\mathrm{F}$ & $\mathrm{F}$ & $\mathrm{F}$ \\
\hline Nunca & 0 & 0 & 0 & 0 & 0 & 0 \\
\hline Casi nunca & 1 & 0 & 0 & 0 & 0 & 0 \\
\hline A veces & 5 & 1 & 6 & 1 & 8 & 1 \\
\hline Casi siempre & 8 & 8 & 8 & 6 & 5 & 9 \\
\hline Siempre & 6 & 11 & 6 & 13 & 7 & 10 \\
\hline Total & 20 & 20 & 20 & 20 & 20 & 20 \\
\hline
\end{tabular}

Fuente: Elaboración propia de los autores

Tabla 2

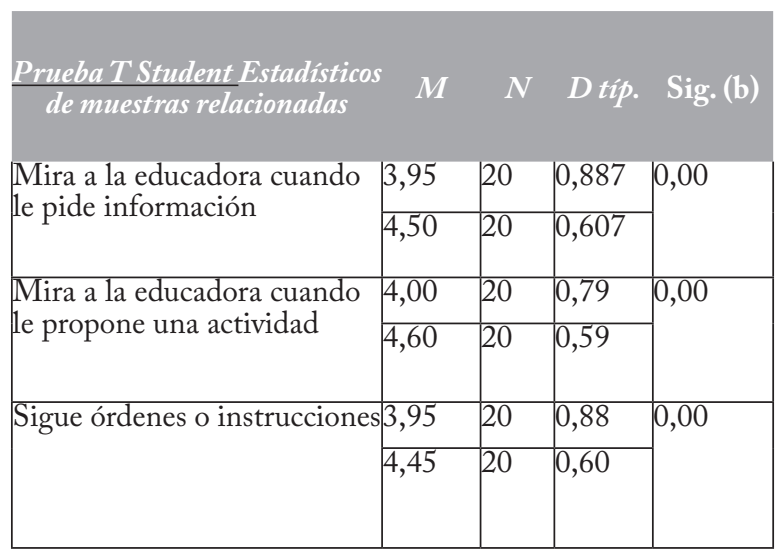

Fuente: Elaboración propia de los autores
En cuanto a la atención en la prueba $\mathrm{T}$ (tabla 2), el nivel de significancia es inferior $\alpha=0,05(0,00<0,05)$ en los indicadores "mira a la educadora cuando le pide información", "mira a la educadora cuando le propone una actividad" y "sigue órdenes o instrucciones", lo cual señala que existen diferencias significativas entre la evaluación diagnóstica y final.

En el primer indicador siempre "mira a la educadora cuando le pide información", de 6 pasó a 11 niños, casi siempre se mantuvo en 8 , a veces de 5 disminuyó a 1 y casi nunca de 1 bajo a ninguno (tabla 1 ). Esto indica que el contacto visual y la interacción que se genera entre la educadora y el niño tendrá influencias en tanto son modelos a seguir por los niños, pero la mayoría de sus aprendizajes los obtienen a través de la observación, así que en la medida que los modelos a seguir sean estratégicos, piensen al actuar, planifiquen o evidencien sus mecanismos de autorregulación, el niño cuando se enfrente a la tarea, juego o actividad lo hará de manera similar (Thornton, 2000).

En el segundo indicador "mira a la educadora cuando le propone una actividad" siempre de 6 pasó a 13 niños, casi siempre de 8 disminuyó a 6 y a veces de 6 disminuyó a 1 (tabla 1), lo cual da a conocer la importancia que tiene el uso de las estrategias didácticas que se ofrecen al momento de realizar una actividad para motivar la atención de los niños, desarrollando la capacidad de aprender a aprender y esto permite precisar los procesos que facilitan el logro de la autonomía y el control en relación con el propio aprendizaje (Lacasa y Herranz, 1995). Por ejemplo, en el juego "Juanito el dormilón", se invitó a los niños a imaginar que estaban en el campo cuidando ovejas; para esto se les narró la historia de Juanito dormilón, quien no se daba cuenta que se le desaparecían sus ovejas cuando se quedaba dormido; entonces se solicitó a un voluntario para que fuera Juanito, se le pidió que observara y vigilara el rebaño, pero este se quedó dormido (se le pidió que saliera del aula), y al despertar, en ocasiones se le perdieron o aparecieron ovejas cuando Juanito entraba al aula y verificaba si estaban todos sus animales... y así sucesivamente se jugó hasta que todos los niños cumplieron con el rol de Juanito dormilón. Frente a esta experiencia, la mirada de los niños estaba centrada en la educadora y en el rebaño de ovejas; si bien no todos lograron el objetivo al dar sus respuestas, sí se logró obtener la concentración y atención en el juego. 
Estos resultados confirman la importancia de motivar la atención: la relación de los niños con la educadora favorece la atención, puesto que la confianza, el buen trato y la orientación adecuada de ella conllevaron a que el desarrollo de los procesos de resolución de problemas fluyera de manera natural por medio de juegos infantiles.

En el tercer indicador "sigue órdenes o instrucciones", siempre 7 aumentó a 10 niños, casi siempre de 5 aumentó a 9 y a veces de 8 disminuyó a 1 (tabla 1). Estos resultados dejan de manifiesto que, en los procesos del desarrollo del pensamiento, "el niño va creando y madurando las estructuras de razonamiento lógico matemático gracias a las interacciones constantes con las personas y el medio que le rodean" (Alsina, 2006, p. 27), es decir, para poder realizar un juego o una actividad deben seguir las órdenes paso a paso para poder lograr el objetivo y el punto de partida para un trabajo formativo (Gilb, 2003). La atención fue mejorando mediante los distintos juegos que les permitieron una mejor comprensión y desarrollo del pensamiento lógico.

Cuando se realizaban juegos activos como "El lagarto Splach", antes de comenzar a jugar primero se les indicaban las reglas del mismo, luego se les pidió que hicieran un círculo eligiendo un voluntario para que fuese el "Lagarto Splach". Este tuvo la labor de atrapar a los monitos traviesos cuando terminaba la canción, pero no podía atrapar a los monitos que se encontraban escondidos detrás de los árboles, quienes eran niños voluntarios. Una vez que atrapó a todos los monitos sueltos los contó y escribió el número que correspondía en el paleógrafo. Para el éxito del juego los niños siguieron las órdenes. La primera vez que jugaron los niños se mostraron muy entusiastas, aunque les costó seguir las órdenes, puesto que todos querían correr y ser atrapados; si bien todos contaron correctamente la cantidad de monitos atrapados, solo 4 niños contaron uno a uno y el total lo asociaron con el número correspondiente. La segunda vez que jugaron ya tenían claras las órdenes a seguir, por lo que el juego resultó exitoso, aumentando a 6 niños la asociación de número -cantidad. En posteriores veces de la repetición del juego fue aumentando la cantidad de niños que podían seguir las órdenes y contar uno a uno los niños atrapados.

Los procesos de aprendizaje, dependiendo de donde se aborden, requieren de procesos como la atención, almacenaje y memoria, el paradigma cognitivo que lo define como aprendizaje guiado. El paradigma constructivo lo plantea como mediador o proceso constructivo, que incluye evaluación, transferencia, entre otros.

Monereo y Castelló (1997), establecen que las estrategias son "procesos de toma de decisiones que se realizan conscientemente para alcanzar un objetivo, en los que activan técnicas y procedimientos de distinta naturaleza (disciplinarios e interdisciplinarios)" (p. 78). Es así que la atención es una función cognitiva que madura a lo largo del desarrollo, al igual que el control de los impulsos que involucra los mecanismos de selección, distribución y mantenimiento de la actividad psicológica (León, Jiménez y Restrepo, 2010), a través de estrategias donde se requiere de planificación, reflexión, evaluación. Mediante estos procesos algunos niños pudieron ser capaces de recuperar las estrategias que les sirvieron en otras situaciones y saber qué procedimiento utilizaron según sea las tareas, bien para repetir acciones, situaciones o para resolver problemas.

\section{COMPRENSIÓN}

Tabla 3

\begin{tabular}{|c|c|c|c|c|c|c|}
\hline \multicolumn{3}{|c|}{$\begin{array}{c}\text { Comprende las tareas } \\
\text { que se le proponen }\end{array}$} & \multicolumn{2}{|c|}{$\begin{array}{c}\text { Tiene } \\
\text { problemas en } \\
\text { la compren- } \\
\text { sión de los } \\
\text { términos que } \\
\text { se utilizan }\end{array}$} & \multicolumn{2}{|c|}{$\begin{array}{l}\text { Aunque ver- } \\
\text { balmente no } \\
\text { comprenda una } \\
\text { tarea, lo bace } \\
\text { cuando el profe- } \\
\text { sor le bace una } \\
\text { demostración. }\end{array}$} \\
\hline & $\mathrm{D}$ & $\mathrm{Ef}$ & $\mathrm{D}$ & E f & $\mathrm{D}$ & $\mathrm{E} \mathrm{f}$ \\
\hline & $\mathrm{F}$ & $\mathrm{F}$ & $\mathrm{F}$ & $\mathrm{F}$ & $\mathrm{F}$ & $\mathrm{F}$ \\
\hline Nunca & 0 & 0 & 0 & 1 & 1 & 0 \\
\hline Casi nunca & 1 & 0 & 7 & 12 & 5 & 5 \\
\hline A veces & 9 & 2 & 10 & 7 & 4 & 0 \\
\hline Casi siempre & 5 & 10 & 3 & 0 & 8 & 9 \\
\hline Siempre & 5 & 8 & 0 & 0 & 2 & 6 \\
\hline Total & 20 & 20 & 20 & 20 & 20 & 20 \\
\hline
\end{tabular}

Fuente: Elaboración propia de los autores

I Panorama I pp. 98-107 I Volumen 10 I Número 19 
Tabla 4

\begin{tabular}{|c|c|c|c|c|c|}
\hline & 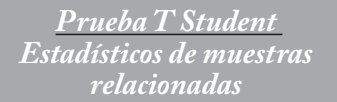 & $M$ & $N$ & $\begin{array}{c}\mathrm{D} \\
\text { tip. }\end{array}$ & Sig. (b) \\
\hline Ana Patricia & \multirow{2}{*}{$\begin{array}{l}\text { Comprende las tareas } \\
\text { que se le proponen. }\end{array}$} & 3,70 & 20 & 0,92 & \multirow[b]{2}{*}{0,00} \\
\hline $\begin{array}{l}\text { León Urquijo I } \\
\text { Jacqueline del }\end{array}$ & & 4,30 & 20 & 0,66 & \\
\hline Carmen Casas & \multirow[b]{2}{*}{$\begin{array}{l}\text { Tiene problemas en } \\
\text { la comprensión de los } \\
\text { términos que se utilizan }\end{array}$} & 2,800 & 20 & 0,70 & \multirow[b]{2}{*}{0,014} \\
\hline $\begin{array}{l}\text { Antilef I } \\
\text { Gerardo }\end{array}$ & & 2,30 & 20 & 0,57 & \\
\hline Restrepo & \multirow[b]{2}{*}{$\begin{array}{l}\text { Aunque verbalmente no } \\
\text { comprenda una tarea lo } \\
\text { hace cuando el profesor } \\
\text { le hace una demostración }\end{array}$} & 3,25 & 20 & 1,11 & \multirow[b]{2}{*}{0,086} \\
\hline Ramírez I & & 3,80 & 20 & 1,15 & \\
\hline
\end{tabular}

Fuente: Elaboración propia de los autores

En la comprensión, los resultados de la prueba $\mathrm{T}$ (tabla 4), el nivel de significancia es inferior $\alpha=0,05(0,00$ $<0,05$ y $0,014<0.05$ ) en los dos primeros indicadores "comprende las tareas que se le proponen" y "tiene problemas en la comprensión de los términos que se utilizan" respectivamente, existe diferencia significativa entre el diagnóstico y la evaluación final. En el indicador "aunque verbalmente no comprenda una tarea, lo hace cuando el profesor le hace una demostración" el nivel significancia es mayor a $\alpha=0,05(0,086>$ a 0,05$)$, lo que indica que no existe diferencia significativa entre el diagnóstico y la evaluación final.

En el primer indicador "comprende las tareas que se le proponen", el indicador siempre 5 aumentó a 8 niños, casi siempre de 5 aumentó a 10, y a veces de 9 disminuyó a 2 (tabla 3), porque se encontraron en el estado de elaboración del pensamiento de la comprensión de situaciones que se les proponen. Las posibilidades de aprendizaje y desarrollo dependen de la zona de desarrollo próximo (ZDP), y el estímulo para llegar a la zona del desarrollo potencial, que se crean espacios que generen la inte-

Cuando se realizó el juego "Enceste de cartas", los niños debían encestar cartas en diferentes cajas; para esto se formaron cuatro equipos con un color para cada uno (amarillo, rojo, azul y verde). Cada equipo se sentó en filas y se demarcó una línea sobre el suelo en la que debían pararse al momento de lanzar. Cada niño tenía tres cartas, La educadora les dijo que cuando todos lanzaran las cartas a la cesta, el primer niño de cada fila debía contar cuántas cartas encestó su equipo, la cantidad sería escrita por ella en la pizarra, para luego preguntarles qué equipo había encestado más cartas. E1 mismo juego se realizó cambiando de lugar los equipos y se sumaron los resultados a los anteriores; la educadora, junto con los niños decidieron qué grupo había encestado más cartas. Además, se les preguntó por qué ganaban la cantidad más alta; la respuesta de un niño fue - "porque hay más", la educadora le pregunta -“jpor qué hay más?", respondiendo _ "porque es un número grande (9) y en la caja hay nueve cartas". De esta forma se puede comprender que la actividad matemática se fundamenta en el reconocimiento de las competencias numéricas tempranas, en las representaciones, procedimientos y en el progresivo incremento de la comprensión (Orozco y Otálora, 2003).

En este juego, las órdenes entregadas fueron recibidas correctamente por la mayoría de los niños; a quienes no entendían se les explicó de manera más personalizada, pero a medida que pasaban los niños fueron comprendiendo de mejor manera el juego. Al momento de contar las cartas todos dieron a conocer la cantidad, puesto que eran cantidades bajas, se prestó ayuda solo a tres niños. Cuando se sumó las cartas de cada grupo, solo tres niños acertaron a la cantidad correcta de todos los grupos, cuatro acertaron solo a las cantidades bajas y el resto de los niños imitaban sus respuestas dándolas a conocer a viva voz. Después se les pregunto nuevamente qué equipo había ganado, y todos contestaron _ "el que tiene 9," y se les preguntó por qué, la respuesta de un niño fue _ "porque tiene muchos", a esta respuesta se sumó un niño quien afirmó la respuesta.

Para lograr que los niños fueran capaces de darse cuenta de qué conocimientos necesitaban para que les resultara fácil un juego o una tarea, debieron recurrir a aplicar diversas estrategias de resolución de problemas, pues "es importante considerar el 'análisis del error' como elemento relevante (Sáiz, 2000); ya que estudiando dónde estriba el fallo se puede redirigir la resolución hacia un proceso correcto facilitando la construcción del conocimiento" (Sáiz y Roman, 2011, p. 11).

En el indicador "tiene problemas en la comprensión de los términos que se utilizan", la categoría nunca de 0 pasó a 1 
que presentaban dificultad en la comprensión de términos; casi nunca de 7 aumentó a 12; a veces de 10 disminuyó a 7 (tabla 3). Cada vez que los niños presentaban problemas en la comprensión de los juegos o actividades se les daba la oportunidad de expresar cuáles eran sus dificultades en la tarea. Frente a esto, Orozco y Otálora (2003) afirman que se debe propiciar que los niños confronten, evalúen y retroalimenten sus conocimientos, lo que les permite construir procedimientos más sofisticados y conceptos con mayores niveles de abstracción.

Muchas veces los niños que tenían problemas en la comprensión lo que hacían era manifestar la dificultad a la hora de resolver problemas; según Thornton (2000), esto puede ser por la limitación de los conocimientos previos que posean sobre las tareas, actividades o juegos a los que se enfrenten.

En este estudio, los niños comenzaron a trabajar desde abril con el programa educativo "Jugando con las matemáticas”, lo que sin duda reflejó el aumento de la información que ellos poseían en cuanto al desarrollo de la resolución de problemas y el pensamiento lógico, observándose la individualidad de cada uno, pues el conocimiento que fueron adquiriendo crecía a su propio ritmo.

En el indicador "aunque verbalmente no comprenda una tarea, lo hace cuando el profesor le hace una demostración", los cambios entre el diagnóstico y la evaluación final fueron pocos, siempre de 2 pasó a 6 niños, casi siempre de 8 pasó a 9 , casi nunca de 5 quedó igual y nunca de 1 disminuyó a ninguno. Aunque seis de los niños lograron realizar tareas abstractas, como por ejemplo resolver problemas de enunciado verbal en los que se les invito a concentrarse y escuchar muy atentamente las preguntas, antes se les explicó que solo podría responder el niño que levantara la mano: las preguntas fueron las siguientes: - ¿Cuántas patas tienen 2 leones? ¿Cuántas orejas tienen 3 conejos? ¿Cuántas ruedas tienen 2 autos? ¿Cuántas manos tienen 2 niños? ¿Cuántas patas tienen 3 pollos?

En esta experiencia lo primero que comenzaron a hacer los niños fue adivinar la cantidad, diciendo números al azar, pero se observó a cuatro niños en particular quienes se quedaban pensando y analizando la pregunta; tres de ellos llegaron a la solución contando con sus dedos en el aire o con sus dedos uno a uno imaginando las orejas de los conejos, dando como respuesta 5, luego dicen - " $n o$, no..." vuelven a contar con sus dedos y dicen 6 , logrando la respuesta correcta; el resto de los niños que logró el resultado de las preguntas lo hicieron contando con sus dedos imitando a sus pares. A la pregunta “¿cuántas ruedas tienen dos carros?”, solo un niño pudo resolverlo: se agachó y miró debajo de las mesas, luego dijo - "tienen 8 ruedas"; cuando se le preguntó por qué sabía, dijo: “como un auto tiene 4 ruedas y la mesa 4 patas, dos mesas tienen 8 patas y dos autos tienen 8 ruedas".

La educadora es mediadora, siempre debe entregar la ayuda necesaria y ajustarla cuando los niños necesiten, ya que muchas veces una intervención poco acertada puede inhibir el uso de estrategias y la realización de tareas, juegos o actividades. (Thornton, 2000).

Cuando finalizó la ronda de preguntas y respuestas se revisó conjuntamente las respuestas, verificando de manera concreta los resultados, pudiéndose notar que los niños que no pudieron hacerlo en forma abstracta a través de las preguntas, comprendieron cuando se realizó de manera concreta o tras un modelaje de cada actividad.

Dentro de la resolución de problemas se encuentran los problemas de enunciado verbal, los que requieren de un análisis de la incógnita, pueden contar con demasiados o pocos datos, se pueden resolver de más de una manera y "los problemas de enunciado no verbal son los que tienen como soporte principal la imagen: los problemas visuales y los problemas de secuencia” (Alsina, 2006, p. 142). Muchas veces los niños manifiestan dificultades a la hora de enfrentarse a los problemas y esto puede ser por la limitación de los conocimientos previos que poseen sobre las tareas, actividades y problemas a los que se vean enfrentados.

Existen otros factores como la capacidad que tienen los niños "para hacer inferencias correctas a partir de la representación propia que se hacen del problema y la dificultad para aprender adecuadamente la información que se requiere y que influye de manera directa en tratar de encontrar la solución correcta" (Thornton 2000 , p. 80), por lo que sin duda las habilidades para la resolución de problemas dependen del aumento de la información que posean los niños, pues el conocimiento que ellos van adquiriendo a su propio ritmo y sus destrezas les ayudará a comprender los procedimientos que conlleva realizar procesos metacognitivos propios del desarrollo del pensamiento lógico.
| Desarrollo del

pensamiento

lógico basado

en resolución de problemas en niños de 4 a 5 años 
MOTIVACIÓN

Tabla 5

\begin{tabular}{|c|c|c|c|c|c|}
\hline Ana Patricia & \multicolumn{3}{|c|}{$\begin{array}{l}\text { Tiene interés hacia las tareas que se } \\
\text { le proponen }\end{array}$} & \multicolumn{2}{|c|}{$\begin{array}{l}\text { El interés depende del } \\
\text { tipo de tarea }\end{array}$} \\
\hline Jacqueline del & & $\mathrm{D}$ & $\mathrm{Ef}$ & $\mathrm{D}$ & $\mathrm{Ef}$ \\
\hline Carmen Casas & & $\mathrm{F}$ & $\mathrm{F}$ & $\mathrm{F}$ & $\mathrm{F}$ \\
\hline Antilef I & Nunca & 0 & 0 & 1 & 2 \\
\hline Gerardo & Casi nunca & 0 & 0 & 7 & 7 \\
\hline Restrepo & A veces & 6 & 2 & 8 & 9 \\
\hline Ramírez I & Casi siempre & 8 & 6 & 4 & 1 \\
\hline & Siempre & 6 & 12 & 0 & 1 \\
\hline & Total & 20 & 20 & 20 & 20 \\
\hline
\end{tabular}

Fuente: Elaboración propia de los autores

Tabla 6

\begin{tabular}{|c|c|c|c|c|}
\hline$\frac{\text { Prueba T Student Estadisticos }}{\text { de muestras relacionadas }}$ & $M$ & $N$ & $\begin{array}{l}\mathrm{D} \\
\text { tip. }\end{array}$ & Sig. (b) \\
\hline \multirow[b]{2}{*}{$\begin{array}{c}\text { Tiene interés hacia las tareas } \\
\text { que se le proponen }\end{array}$} & 4,00 & 20 & 0,79 & \multirow[b]{2}{*}{0,002} \\
\hline & 4,50 & 20 & 0,68 & \\
\hline \multirow{2}{*}{$\begin{array}{l}\text { El interés depende del tipo } \\
\text { de tarea }\end{array}$} & 2,75 & 20 & 0,85 & \multirow[b]{2}{*}{0,419} \\
\hline & 2,60 & 20 & 0,94 & \\
\hline
\end{tabular}

Fuente: Elaboración propia de los autores

En el ítem de motivación en el primer indicador "Tiene interés hacia las tareas que se le proponen", los resultados de la prueba $\mathrm{T}$ (tabla 6 ), el nivel de significancia es inferior $\alpha=0,05(0,002<0,05)$, existe diferencia significativa entre el diagnóstico y la evaluación final, ya que siempre de 6 aumentó a 12 niños que muestran interés, casi siempre disminuyó de 8 a 6 , y a veces de 6 pasó a 2 (tabla 5). Si bien el interés de los niños por realizar actividades o juegos fue aumentando, puesto que cada vez

Panorama I pp. 98-107| Volumen 10 I Número 19 | Julio-diciembre 2016 | comprendían de mejor manera, sumado a esto las ganas de hacerlo bien o ganar, iban mejorando sus competencias; además, la complejidad de los juegos hizo que cada vez hubiese nuevos restos, porque las tareas cuando son demasiado fáciles hacen que se pierda el interés en ellas y no se transformen en problemas (Thornton, 2000). Demostraron mayor interés y persistencia cuando armaron un "Puzle de un cuadrado", de cinco fichas de papel, y los pegaron cuando terminaron de armarlo. Se les brindó apoyo verbal y motivación para que lo intentaran las veces que fuera necesario, se observó a los niños intentar encajar los polígonos una y otra vez sin poder armarlo, pedían ayuda o decían que no lo podían hacer, argumentando que era muy difícil; a los 10 minutos de iniciada la actividad una niña lo logró, dos minutos más tarde un niño lo hizo con un poco de ayuda de la educadora ya que una pieza estaba al revés, y de 18 niños restantes solo 2 adquirieron la competencia. En la evaluación final para armar el puzle de un triángulo, de los 20 niños lo lograron 7 en 10 minutos, los demás siguieron intentando sin perder la motivación hasta que lo lograron en mayor tiempo.

El conocimiento de la tarea permitió que los niños usaran algunas estrategias cognitivas y/o metacognitivas en la resolución de problemas; esas estrategias fueron adecuadas para lograr los objetivos deseados, puesto que de esta manera tomaron conciencia de las mejores estrategias de resolución para cada situación a la que se había enfrentado.

La resolución de problemas juega un papel muy importante en la motivación para el desarrollo del pensamiento lógico, ya que está presente en todo momento bien sea en sus juegos, en su mundo social o en su quehacer diario. El ser humano en cualquier etapa de su vida tiene la posibilidad de resolver problemas (Thornton, 2000). En la medida que los niños tuvieron más información y experiencias pudieron adquirir más estrategias para resolver los problemas.

La experiencia tiene un peso importante en el proceso de resolución de problemas; tal es así que dos niños que utilizaron el mismo proceso para resolver un problema pudieron llegar a conclusiones diferentes, dependiendo de las experiencias de cada uno. A esto se suma que a partir de la experiencia se obtuvo mayor información y cuanta más rica esta sea, más fácil será para el niño saber cómo abordar un problema (Thornton, 2000).

También es importante tener en cuenta que por más que los niños lograron la experticia en algunas tareas, no fue esta una condicionante para que lo fuese en otras, ya que los procesos no se generalizan a todas las áreas. Otro de los elementos que jugó un papel importante fue cómo se enfrentaban a las tareas, puesto que influye el autoconcepto, ligado a la experiencia social y a la posición que ocupa dentro del entorno (Thornton, 2000). En este sentido, la interacción con la educadora se convirtió en una influencia importante, toda vez que ella fue modelo 
a seguir por los niños. Si bien es verdad que los niños no solo aprendieron de sus modelos, gran parte de sus aprendizajes los obtuvieron de la observación y la imitación, así es que en la medida que ella fue estratégica, pensó para actuar, planificó o evidenció sus mecanismos de autorregulación, los niños cuando se enfrentaron a las tareas o problemas lo hacían de manera similar. La educadora en la interacción con los niños también fue mediadora, proporcionando siempre la ayuda necesaria y ajustándola cuando ellos lo requirieron.

En el indicativo si "el interés depende del tipo de tarea", los resultados de la prueba $T$ (tabla 6) el nivel de significancia es superior $\alpha=0,05(0,419>0,05)$, no existe diferencia significativa entre el diagnóstico y la evaluación final, puesto que en esta ocasión el interés por la tarea fue casi el mismo durante el desarrollo de los espacios de interacción y en los dos momentos de evaluación (tabla 5), además que la metodología del trabajo se centró en el juego educativo, que no es solo divertir, sino más bien extraer sus enseñanzas (Ferrero, 2004), siendo el juego una gran estrategia didáctica para desarrollar con niños el interés por las actividades y/o tareas, puesto que los materiales que permiten la interacción como rompecabezas, rondas, cantos, bingos, laberintos entre otros, permitieron captar la atención y el interés de los niños estimuló el desarrollo del pensamiento a través de aplicaciones prácticas y entretenidas para ellos, por tanto en este estudio los niños casi nunca perdieron el interés en participar de manera concreta o abstracta, consiguiendo generar en ellos el punto de partida para la enseñanza de la matemática.

Ante todo, la actividad matemática debe fundamentarse en el reconocimiento de las competencias numéricas tempranas, tanto a nivel de sus representaciones, como de sus procedimientos y en el progresivo incremento de la comprensión. Si los maestros son conscientes de la gama de habilidades que un niño posee antes de los 6 años, edad en la que empieza su educación básica primaria, pueden aprovecharlas para iniciar su transformación hacia un conocimiento propiamente matemático (Orozco y Otálora, 2003, p. 202).

En el aula, la resolución de problemas matemáticos que incluyó la educadora permitió mantener el interés de los niños para enfrentarse a situaciones problemas con actividades motivadoras en función de los contenidos que se quiso trabajar.
Fue importante dar la oportunidad a los niños de expresarse y de dar a conocer cuáles fueron sus competencias cuando se enfrentaron a situaciones problemas. Según Monereo (2007), se logra formar la autonomía en los niños a través de la posibilidad de autorregular en ellos el propio aprendizaje, gracias a la mediación de otros, dando mayores frutos en orden a lograr el propósito de la formación de niños que utilicen las estrategias para resolver problemas. "Los métodos que se basan en la reflexión en voz alta y la gradual cesión de las decisiones que deben tomarse en la resolución de problemas, han resultado especialmente beneficiosos" (p. 505).

Cuando los niños realizaban la reflexión consciente al explicarse el significado de los problemas a resolver y al tomar decisiones sobre su posible resolución, fue una especie de diálogo consigo mismo. Así, cuando empleaban una estrategia fueron conscientes de sus propósitos y, cuando se desviaban de ellos, fueron capaces de reorientar o regular sus acciones (Monereo, Castelló, Clariana, Palma y López, 2007).

Frente a esto fue necesario promover diversas fases para el desarrollo de la resolución de problemas, siendo la primera el diagnóstico, estableciendo en qué etapa de conocimiento y habilidades se encontraban los niños al momento de iniciar el estudio, pues así se obtuvo un análisis previo y el punto de partida para poder realizar la intervención. En esta fase "se pretende que el niño confronte, evalúe y retro-alimente su conocimiento, construyendo procedimientos más sofisticados y conceptos con mayores niveles de abstracción" (Orozco y Otálora 2003, p. 205). En la fase del seguimiento, la cual incorporó las dos fases antes mencionadas demostrando la continuidad en el tiempo, permitió a la educadora seguir los procesos de aprendizaje que van desarrollando los niños, pudiendo verificar los avances o retrocesos que se pudieron generar. He aquí el rol mediador de la educadora que se encontró presente, puesto que, al observar detenidamente los procesos en la resolución de problemas de los niños, fue necesario la interacción entre ellos para compartir experiencias.

Es así como el éxito de resolver problemas dependió de los tipos de inferencias que los niños pudieron realizar, los aspectos que lograron extraer tanto de tareas, juegos, experimentos, entre otros, reconociendo y comprendiendo con la ayuda de las estrategias de juego y ensayo-error que facilitaron a la resolución de problemas a
| Desarrollo del pensamiento

lógico basado en resolución de problemas en niños de 4 a 5 años 
medida que fueron adquiriendo experiencias y conocimiento sobre los problemas planteados.

\section{CONCLUSIONES}

Ana Patricia

León Urquijo I

Jacqueline del

Carmen Casas

Antilef I

Gerardo

Restrepo

Ramírez |

En este estudio se encontró que la atención es la más favorecida en los niños gracias a su relación con la educadora, el trato de confianza, instrucciones correctas y bien dirigidas, uso del lenguaje adecuado y orientación apropiada, conllevando al mayor progreso de ellos en la resolución de problemas.

$\mathrm{El}$ interés por las tareas también mejoró de forma significativa en los niños, puesto que las estrategias didácticas interesantes y motivadoras, además de llamar su atención les permitió mayor concentración.

En cuanto a lograr explicar verbalmente si las tareas les resultaron fáciles o difíciles, es importante señalar que los principios relacionados con el aprendizaje y el proceso del desarrollo del pensamiento mediante la resolución de problemas en la primera infancia -sin olvidar que los niños poseen la capacidad de resolver problemas- se fueron desarrollando a través del tiempo con la adquisición de nuevas experiencias y aprendizajes; estos se pulieron por medio de modelos significativos que se encontraron en su contexto familiar, social y escolar. Es así que demostraron destreza cuando resolvieron problemas de enunciado verbal, comprendieron las tareas a realizar, y cuando las respuestas fueron erróneas buscaron alternativas realizando una evaluación de su procedimiento mediante el razonamiento.

Estas experiencias pasadas a la resolución de problemas en tareas cotidianas, como cuantas llantas tienen dos autos, con el uso del lenguaje, les permitió realizar asociaciones de número-cantidad de manera divertida por medio de juegos continuos, adquiriendo destrezas y Panorama I habilidades cognitivas junto a la experticia que han ido pp. 98-107| obteniendo a lo largo de sus vidas.

Volumen 10 I Número 19। Julio-diciembre 2016 । 106
Se observa bastante mejoría en este grupo de niños en la comprensión de los problemas a resolver. Aquí es importante resaltar que para los niños que mejoraron su capacidad de resolución de problemas, esto contribuyó al desarrollo del pensamiento lógico, no solo mediante el aprendizaje de variadas estrategias, sino que además lograron ser conscientes de la manera de solucionar las situaciones planteadas y que esta resultara significativa para su desarrollo cognitivo; del mismo modo, fueron capaces de reconocer que una situación tiene determinadas características y pasos a seguir para el logro de competencias que pudieron utilizar para resolver otros problemas en forma parcial o total, y comprendieron que otros problemas requieren otro tipo de estrategias diferentes. Descrito así parece muy complejo, pero los niños, en esta etapa del desarrollo pensamiento intuitivo, van adquiriendo con oportunidades de interacción sobre los objetos, con finalidades específicas, funciones lógicas del pensamiento que les permitieron la agilidad mental para encontrar soluciones a otros problemas que se les presentan en el ambiente escolar y en la vida cotidiana.

Resolver un nuevo problema fue una tarea intelectual estimulante para los niños, ya que los impulsó a reflexionar desarrollando la capacidad de pensar, valorando sus propios procesos de aprendizaje, descubriendo nuevos conceptos e inventando nuevas estrategias para solucionar sus problemas, lo que dio como resultado niños más conscientes de sus posibilidades y con un mejor desarrollo cognitivo que los estimuló a pensar, experimentar y autorregularse.

Por otra parte, la educadora fue la facilitadora de estrategias de juegos que los niños necesitaban para encontrar las soluciones que les llevarían a resolver problemas, tuvo gran importancia la comunicación clara de las instrucciones de la tarea o actividad y evitó en lo posible dar soluciones inmediatas; más bien guio a los niños para que experimentaran y pusieran en práctica la resolución del problema, pues la idea era que tuvieran la oportunidad para demostrar su capacidad de reflexión y encontrar sus propias estrategias para resolverlo, ampliando su capacidad de pensar y sobre todo su capacidad de buscar y escoger el camino a seguir para solucionarlo, y cuando lo encontraron sintieron la satisfacción de haberlo realizado.

La resolución correcta de los problemas depende de la experiencia concreta y de la familiaridad de la tarea a realizar, siendo esta la que aumente la capacidad de razonar, ya que los niños recurren a conocimientos previos de su mundo real y son capaces de extrapolar esos hallazgos a nuevas situaciones. 
El juego fue indudablemente el mejor aliado como estrategia didáctica para la resolución de problemas, puesto que de forma divertida los niños aprendieron de una acción propia de su condición infantil -que muchas veces se pierde a través de la evolución educativa, por considerarla poco seria-, pero que la educadora hizo buen uso de ella, consiguió aprendizajes duraderos que generaron otros, dentro de la cadena interminable del desarrollo del pensamiento lógico.

La motivación y la satisfacción de los logros en la resolución de problemas estimuló a los niños a continuar con nuevas tareas, haciendo que el desarrollo del pensamiento lógico se preparara para la etapa escolar posterior, donde tendrán que enfrentarse al aprendizaje de la matemática que ha sido tradicionalmente el área de mayor dificultad, más por una condición social, no porque realmente lo sea.

Los niños de 4 a 5 años no solamente resolvieron problemas en acción sobre los objetos, también lo hicieron por medio de la abstracción, porque son capaces de dar una respuesta en forma oral que han elaborado a partir de la reflexión sobre las situaciones planteadas, donde tuvieron que hacer uso de los aprendizajes anteriores realizados en forma concreta.

Mediante los instrumentos utilizados en este estudio, con las comparaciones y análisis de las notas de campo, se pudo encontrar que algunos niños son más recursivos en la resolución de situaciones problemas con las instrucciones planteadas; lo más habitual fue pedir ayuda cuando lo requirieron, se les repitió las instrucciones y se les proporcionó estímulos de apoyo que les ayudó a lograrlo, algunas veces por sí solos de acuerdo con sus percepciones y habilidades, observando cómo lo hacían otros niños, interactuando y ayudándose entre ellos. Esto reafirmó que el desarrollo del pensamiento se propició en un ambiente social, en la interacción con otros haciendo uso de la comunicación y apoyo mutuo.

\section{REFERENCIAS}

1. Alsina, Á. (2006). Cómo desarrollar el pensamiento matemático de 0 a 6 años. Barcelona: Editorial Octaedro.

2. Ferrero, L. (2004). Eljuego y la matemática. Madrid: Editorial La Muralla, S.A.
3. Gelman, R.y Gallistel, C. R. (1978). The Child's understanding number. Cambridge: Harvard University Press.

4. Gilb, S. (2003). Juegos para escolares. México: Editorial Pax México. S. A

5. Hernández, R. Fernández, C.y Baptista, P. (2010). Metodología de la investigación. México, D.F: Editorial Mc Graw-Hill.

6. IBM Corporation. (2011). Guía breve de IBM SPSS Statistics 20. New York: Editorial Copyright IBM.

7. Lacasa, P.y Herranz, P. (1995). Aprendiendo a aprender. Resolver problemas entre iguales. Madrid: Publicaciones del Ministerio de Educación y Ciencia, España.

8. Lacasa, P. y Villuendas, D. (1988). Acción y representación en el niño: interacción social y aprendizaje. Madrid: Ministerio de Educación y Crencia CIDE.

9. León U.,A.P., Jiménez R. A. Ma. y Restrepo R., G. (2010). El trastorno por déficit de atención en el sector educativo oficial de Armenia. Revista UO. 9(5),1-20. Recuperado de: file:///C:/Users/ aleon/Downloads/Dialnet-E1TrastornoPorDefi citDeAtencionEnE1SectorEducativo-3629335. pdf

10. Monereo, C. (2007). Hacia un nuevo paradigma del aprendizaje estratégico: el papel de la mediación social, del self y de las emociones. Revista Electrónica de Investigación Psicoeducativa, 5(3), 497-534.Recuperado el 23-06-2013 de: http:// www.investigacion-psicopedagogica.org/revista/ articulos/13/espannol/Art_13_206.pdf

11. Monereo, C.y Castelló, M. (1997). Las estrategias de aprendizaje. Barcelona: Editorial Edebé.

12. Monereo, C. (coord.). Castelló, M., Clariana, M., C., Palma, M., y López, Ma. L. (2007). Estrategias de enseñanza y aprendizaje. Formación del profesorado y aplicación en la escuela. Barcelona: Editorial Graó.

13. Orozco, M. y Otálora, Y. (2003). Las competencias matemáticas de los niños pequeños. Artes gráficas del Valle Editores-impresores Ltda.

14. Sáiz, Ma. (2000). Entrenamiento metacognitivo en el aula. Un procedimiento curricularmente integrado, en J. N. García S. (coord.) (2000). De la psicología de la instrucción a las necesidades curriculares. Andalucía: Oikos-Tau S. A., 53-64.

15. Sáiz,M. y Román, J. (2011). Entrenamiento metacognitivo y estrategias de resolución de problemas en niños de 5 a 7 años. International Journal of Psychological Research, 4(2), 9-19.

16. Thornton, S. (2000). La resolución infantil de problemas. España: Editorial Morata. 